\title{
The length of the second shortest geodesic
}

\author{
Alexander Nabutovsky and Regina Rotman
}

\begin{abstract}
According to the classical result of J.P. Serre ([S]) any two points on a closed Riemannian manifold can be connected by infinitely many geodesics. The length of a shortest of them trivially does not exceed the diameter $d$ of the manifold. But how long are the shortest remaining geodesics? In this paper we prove that any two points on a closed $n$-dimensional Riemannian manifold can be connected by two distinct geodesics of length $\leq 2 q d \leq 2 n d$, where $q$ is the smallest value of $i$ such that the $i$ th homotopy group of the manifold is nontrivial.
\end{abstract}

Mathematics Subject Classification (2000). 53C23, 53C22, 58E10.

Keywords. Length of geodesics, length functional, curvature-free inequalities in Riemannian geometry

\section{Main result}

Here is the main result of the present paper:

Theorem 1.1. Let $M^{n}$ be a closed $n$-dimensional Riemannian manifold, let d denote the diameter of $M^{n}$, and let $q=\min _{i}\left\{\pi_{i}\left(M^{n}\right) \neq 0\right\}$. Then for each pair of points $x, y \in M^{n}$ there exist at least two distinct geodesics connecting $x$, $y$ of length not exceeding $2 q d(\leq 2 n d)$.

Observe that if $x=y$, then the trivial geodesic is the shortest geodesic connecting $x$ and $y$. In this case our theorem asserts the existence of a geodesic loop of length $\leq 2 d$ based at an arbitrary point $x$ of $M^{n}$. This result is the main result of the paper $[\mathrm{R}]$ of one of the authors. Theorem 1.1 can be viewed as a generalization of this result. Our proof of Theorem 1.1 is heavily reliant on methods of [R]. We would like also to refer the reader to the foundational paper of $\mathrm{M}$. Gromov [G] that was a source of motivation for us and inspired some of the techniques of the present paper. 


\section{Filling of cages}

Let us begin by introducing the following definitions and notations.

Definition 2.1. Let $x, y$ be two points in $M^{n}$. An $m$-cage $c$ based at $x, y$ is a collection of $m$ paths $c_{1}, \ldots, c_{m}$ from $x$ to $y$. (For every $i c_{i}$ is a continuous map of $[0,1]$ into $M^{n}$.) The space $C_{x, y, m}$ of all $m$-cages based at $x$ and $y$ can be identified with the $m$ th power of the space of paths from $x$ to $y$. For every $L$ let $C_{x, y, m}^{L}$ denote the space of all $m$-cages based at $x, y$ such that the length of each of the $m$ paths forming the cage is at most $L$. Further, let $C_{x, y, m}^{L, \bar{L}}$ denote the space of all $m$-cages $c=\left(c_{1}, \ldots, c_{m}\right)$ based at $x, y$ such that the length of $c_{1}$ does not exceed $\bar{L}$, and the length of $c_{i}$ for every $i=2,3, \ldots, m$ does not exceed $L$.

Let $\sigma^{m}=\left[v_{0}, v_{1}, \ldots, v_{m}\right]$ be the standard $m$-dimensional simplex with edges of length one. (Here $v_{0}, v_{1}, \ldots, v_{m}$ are its vertices.) As usual, we use the notation $C\left(\sigma^{m}, M^{n}\right)$ for the space of continuous maps from $\sigma^{m}$ to $M^{n}$. Of course, this space can be identified with the space of continuous maps of the $m$-dimensional ball into $M^{n}$.

Definition 2.2. Let $x, y$ be two points in $M^{n}, L, \bar{L}$ two positive numbers, and $N$ a positive integer. A coherent $N$-filling of $m$-cages based at $x, y$ from $C_{x, y, m}^{L, \bar{L}}$ is a collection of continuous maps $\phi_{m}: C_{x, y, m}^{L, \bar{L}} \rightarrow C\left(\sigma^{m}, M^{n}\right)$ for all $m=1,2, \ldots, N$ with the following properties:

1 . For every $m$ and $m$-cage $c$ the map $\phi_{m}(c): \sigma^{m} \rightarrow M^{n}$ maps the $(m-1)$ dimensional face $\left[v_{1}, \ldots, v_{m}\right]$ of $\sigma^{m}$ into $y$.

2. For every $m$ and every $m$-cage $c=\left(c_{1}, \ldots, c_{m}\right)$ the map $\phi_{m}(c)$ maps each of $m$ one-dimensional simplices $\left[v_{0}, v_{i}\right]$ by the map $c_{i}$. (Here we identify $\left[v_{0}, v_{i}\right]$ with $[0,1]$.) In particular, $v_{0}$ is mapped into $x$, and for every 1-cage $c$ we have $\phi_{1}(c)=c$.

3. (Coherence) For every $m=2,3, \ldots, N$, every $m$-cage $c$ and every $i=$ $1,2, \ldots, m$ the restriction of $\phi_{m}$ to the $(m-1)$-dimensional face $\left[v_{0}, \ldots, v_{i-1}\right.$, $\left.v_{i+1}, \ldots, v_{m}\right]$ of $\sigma_{m}$ coincides with $\phi_{m-1}\left(c_{(i)}\right)$, where $c_{(i)}$ denotes the $(m-1)$ cage $\left(c_{1}, \ldots, c_{i-1}, c_{i+1}, \ldots, c_{m}\right)$.

To explain the meaning of conditions 1 and 2 collapse $\left[v_{1}, \ldots, v_{m}\right]$ into a point. Identify this point with the North pole and $v_{0}$ with the South pole of the ball $D^{m}$. Then $m$ edges $\left[v_{0}, v_{i}\right]$ become $m$ meridians on the sphere bounding this ball. We can view $m$-cages as maps from this collection of $m$ meridians into $M^{n}$. The meaning of conditions 1 and 2 is that one can regard a coherent $N$-filling of an $m$-cage as an 
extension of this map to a map of the whole $m$-ball. This extension must depend continuously on the $m$-cage. The meaning of the coherence condition is that extensions in different dimensions are compatible.

Proposition 2.3. Let $L, \bar{L}$ be positive real numbers such that $\bar{L} \geq L$, and $N a$ positive integer. Let $x, y, z$ be any three points of $M^{n}$ such that the distance between any two of them does not exceed L. Assume that there exists exactly one geodesic between $x$ and $y$ of length $\leq \bar{L}+(2 N-3) L$. (If $x=y$, then this geodesic is the constant geodesic.) Then there exists a coherent $N$-filling of $m$-cages based at $x, z$ from $C_{x, z, m}^{L, \bar{L}}$.

Proof. We present a proof by induction on $N$. Its base corresponds to the case $N=1$. In this case $\phi_{1}(c)=c$. (Recall that each 1-cage is, by definition, a path in $M^{n}$, i.e. a continuous map of $\sigma^{1}=[0,1]$ into $M^{n}$.) The proof of the induction step is based on the following lemma:

Lemma 2.4. Let $\bar{L}, L$ be positive numbers. Assume that $x, y, z$ are three points in $M^{n}$ such that all distances between them do not exceed L. Assume that there exists only one geodesic between $x$ and $y$ of length $\leq \max \{\bar{L}, L\}+L$. Then any two paths $\gamma_{1}, \gamma_{2}$ starting at $x$ and ending at $z$ such that the length of $\gamma_{1}$ is $\leq \bar{L}$ and the length of $\gamma_{2}$ is $\leq L$ can be connected by a path homotopy that passes only through paths of length $\leq \bar{L}+2 L$. This path homotopy depends continuously on $\gamma_{1}$ and $\gamma_{2}$.

Proof. Let $\sigma$ be the unique shortest geodesic from $x$ to $y, \tau$ be one of the shortest geodesics from $z$ to $y$ (see Figure 1). Every path from $x$ to $y$ of length $\leq 2 L$ or $\leq \bar{L}+L$ can be connected to $\sigma$ by a length non-increasing homotopy. (Otherwise, there will be

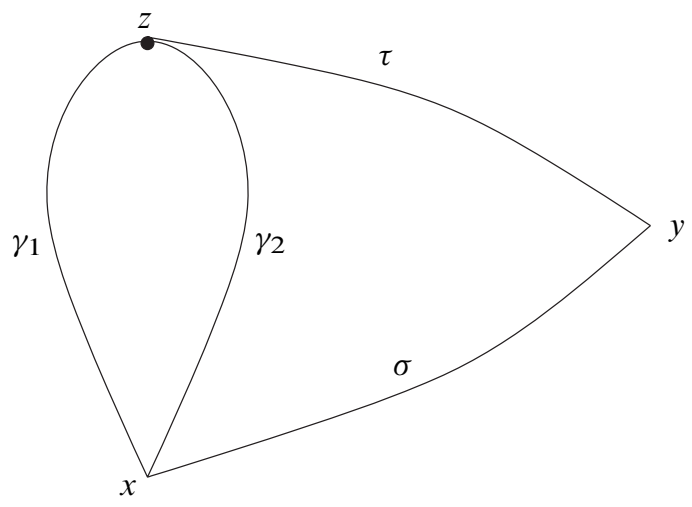

Figure 1 
a second geodesic of length $\leq \max \{\bar{L}, L\}+L$.) Moreover, we can choose a specific length non-increasing homotopy, e.g. the Birkhoff curve-shortening process with fixed endpoints. (See [C] for a detailed description of the Birkhoff curve-shortening process.) This homotopy continuously depends on the initial path. In particular, this homotopy can be used to deform $\gamma_{i} * \tau$ to $\sigma,(i=1,2)$, as well as to deform $\sigma$ back to $\gamma_{i} * \tau$.

Let $\tau^{-1}$ denote the path $\tau$ traversed in the opposite direction. One can construct the desired path homotopy from $\gamma_{1}$ to $\gamma_{2}$ as follows: $\gamma_{1} \rightarrow \gamma_{1} * \tau * \tau^{-1} \rightarrow \sigma * \tau^{-1} \rightarrow$ $\gamma_{2} * \tau * \tau^{-1} \rightarrow \gamma_{2}$. Here arrows denote homotopies. Note that all homotopies depend continuously on $\gamma_{1}$ and $\gamma_{2}$, and that for each of these homotopies the length of paths during the homotopy does not exceed the maximum of lengths of paths at its begining and its end.

Now assume we have constructed the maps $\phi_{1}, \ldots, \phi_{m-1}$. We will next construct $\phi_{m}$. Let $c=\left(c_{1}, c_{2}, \ldots, c_{m}\right)$ be an $m$-net. We need to map $\sigma^{m}=\left[s_{0}, \ldots, s_{m}\right]$ to $M^{n}$. Because of the coherence condition a map $\psi_{m}(c)$ defined as the restriction of $\phi_{m}$ to $\partial \sigma^{m}$ into $M^{n}$ is already prescribed. By virtue of the induction assumption $\psi_{m}$ is a continuous function of $c$. We need only to contract the (map of the) sphere $\psi_{m}(c)$ to a point so that the contracting homotopy depends continuously on $c$. To achieve this goal note that according to Lemma 2.4 there exists a path homotopy between $c_{1}$ and $c_{2}$ that passes through paths $c_{t}, t \in[1,2]$, of length $\leq \tilde{L}=\bar{L}+2 L$ only. (Here we use the fact that $(2 m-3) L \geq L$ for every $m \geq 2$. So, the assumption of Lemma 2.4 about the non-existence of a second short geodesic between $x$ and $y$ follows from a similar assumption in Proposition 2.3.) Consider a 1-parametric family of $m$-cages $c^{(t)}=\left(c_{t}, c_{2}, \ldots, c_{m}\right)$. So, $c^{(1)}=c$ and $c^{(2)}=\left(c_{2}, c_{2}, c_{3}, \ldots, c_{m}\right)$. Note that $c^{(t)} \in C_{x, y, m}^{L, \tilde{L}}$ for every $t$. By virtue of the induction assumption there exists a coherent filling of all $(m-1)$-subcages of $c^{(t)}$ obtained by removal of one of $m$ strands $c_{i}^{(t)}$, and for every $t$ the resulting $m$ maps of $(m-1)$-dimensional simplices can be "glued" to each other into a map $\psi_{m}\left(c^{(t)}\right): \partial \sigma^{m} \rightarrow M^{n}$. Of course, it is important here that $\tilde{L}+(2(m-1)-3) L=\bar{L}+(2 m-3) L$, and so the required assumption about the non-existence of a second geodesic between $x$ and $y$ of length $\leq \tilde{L}+(2(m-1)-3) L$ holds. Thus, one obtains a homotopy $\psi_{m}\left(c^{(t)}\right)$ between $\psi_{m}(c)$ and $\psi_{m}\left(c^{(2)}\right)$.

It remains to show that $\psi_{m}\left(c^{(2)}\right)$ is canonically and, therefore, continuously contractible. (Here we are concerned about the continuity of the contracting homotopy as a function of $c$.) Note that the boundary of $\sigma^{m}$ consists of $(m+1)$ simplices of dimension $(m-1)$. The maps $\phi_{m-1}$ and, thereby, $\psi_{m}$ map two of these faces, namely, faces corresponding to two copies of the $(m-1)$-cage $\left(c_{2}, c_{3}, \ldots, c_{m}\right)$ in an identical way. Together these two cells form a "folded" map of $S^{m-1}$ to $M^{n}$ that factors through the projection of $S^{m-1}$ to the disc $D^{m-1}$. This map is obviously canonically contractible. In order to construct a homotopy of $\psi_{m}\left(c^{(2)}\right)$ to this "folded" map we 
need to "eliminate" the remaining $(m-1)$ maps of $(m-1)$-dimensional faces of $\sigma^{m}$. But one of these maps is constant, and the remaining $(m-2)$ maps correspond to $(m-1)$-cages of the form $\left(c_{2}, c_{2}, \ldots\right)$. Therefore each of these maps is similarly "folded" and can be connected by a canonical homotopy (over its image) to a map of the corresponding face which is a composition of the projection of the considered face to one of its codimension one faces and $\phi_{m-2}\left(\left(c_{2}, c_{3}, \ldots, c_{i-1}, c_{i+1}, \ldots, c_{m}\right)\right)$ for an appropriate $i$. These homotopies eliminate the remaining $m-1$ faces, as desired.

\section{Filling of $(m, \varepsilon)$-umbrellas}

Let $\sigma^{m-1}=\left[s_{1}, \ldots, s_{m}\right]$ denote the standard $(m-1)$-dimensional simplex such that the lenghs of all of its edges are equal to 1 . Let $s_{*}$ denote the center of $\sigma^{m-1}$.

Definition 3.1. An $(m, r)$-umbrella based at $x, y$ consists of a singular $(m-1)$ simplex $\rho: \sigma^{m-1} \rightarrow M^{n}$, a point $x \in M^{n}$ and $m$ paths in $M^{n}$ connecting $x$ with images of the vertices of $\sigma^{m-1}$ under $\rho$ so that $y=\rho\left(s_{*}\right)$, the image of $\rho$ is contained in a metric ball of radius $r$ in $M^{n}$ centered at $y$, and the length of the image of every straight line segment in $\sigma^{m-1}$ under $\rho$ is less than $r$.

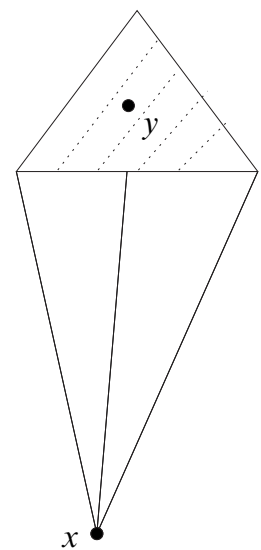

Figure 2. ( $m, r)$-umbrella.

This notion generalizes the notion of $m$-cages that can be considered as $(m, 0)$ umbrellas (with a constant $\rho$ ). The goal of this section is to generalize the notion of coherent filling for $(m, r)$-umbrellas and to extend Theorem 1.1 to $(m, \varepsilon)$-umbrellas for small positive $\varepsilon$. Denote the space of all $(m, r)$-umbrellas based at $x, y$ by $U_{m, r, x, y}$ 
and its subspace formed by all umbrellas where the length of the paths connecting $x$ with the first vertex of $\rho$ does not exceed $\bar{L}$, and the lengths of all paths connecting $x$ with the remaining $m-1$ vertices of the singular simplex $\rho$ do not exceed $L$ by $U_{m, r, x, y}^{L, \bar{L}}$. Each umbrella $u$ can be represented as $\left(c_{1}, \ldots, c_{m}, \rho\right)$, where $c_{i}$ are continuous paths from $x$ to the vertices of the singular simplex $\rho$. It is obvious that 1-umbrellas based at $x, y$ are merely continuous paths starting at $x$ and ending at $y$.

Definition 3.2. Let $N$ be a positive integer and $L, \bar{L}$ positive real numbers. A coherent $N$-filling of $(m, r)$-umbrellas based at $x, y$ from $U_{m, r, x, y}^{L, \bar{L}}$ is a family of continuous maps $\phi_{m}: U_{m, r, x, y}^{L, \bar{L}} \rightarrow C\left(\sigma^{m}, M^{n}\right)$ for $m=1,2, \ldots, N$ such that for every $(m, r)$ umbrella $u=\left(c_{1}, c_{2}, \ldots, c_{m}, \rho\right) \in U_{m, r, x, y}^{L, \bar{L}}$ the following conditions hold:

1. The restriction of $\phi_{m}(u)$ to the $(m-1)$-dimensional face $\left[s_{1}, s_{2}, \ldots, s_{m}\right]$ coincides with $\rho$;

2. The restrictions of $\phi_{m}(u)$ to 1 -dimensional simplices $\left[s_{0} s_{i}\right]$ coincide with $c_{i}$ for $i=1,2, \ldots, m$. In particular, $\phi_{1}(u)=u$ for all 1 -umbrellas $u$;

3. (Coherence) For every $i=1,2, \ldots, m$ the restriction of $\phi_{m}(u)$ to $(m-1)$ dimensional simplex $\left[s_{0}, s_{1}, \ldots, s_{i-1}, s_{i+1}, \ldots, s_{m}\right]$ coinsides with $\phi_{m-1}\left(u_{i}\right)$, where $u_{i}=\left(c_{1}, c_{2}, \ldots, c_{i-1}, c_{i+1}, \ldots, c_{m}, \rho_{i}\right)$, and $\rho_{i}$ is the restriction of $\rho$ to the $(m-2)$-dimensional face of the standard simplex $\sigma^{m-1}$ obtained by exclusion of the $i$ th vertex.

The notion of $(m, \varepsilon)$-umbrellas can be regarded as a generalization of the notion of $m$-cages, where one of the endpoints is being "enlarged" into a small simplex. (If this simplex degenerates into a point, the umbrella becomes a cage.) The next proposition asserts that there exists a generalization of the process of filling of cages described in the proof of Proposition 2.3 to $(m, \varepsilon)$-umbrellas for small $\varepsilon$. The idea of the proof of this generalization is very simple: One can just shrink the small simplex $\rho$ in the definition of umbrellas over itself to a point, thus, obtaining a cage, which then can be filled as in the proof of Proposition 2.3.

Proposition 3.3. Let $L, \bar{L}$ be positive numbers such that $\bar{L} \geq L$. Let $x, y, z$ be any three points in a closed Riemannian manifold $M^{n}$ such that the distance between any two of these three points does not exceed $L$. Let $N>1$ be an integer. Then for every $0<\varepsilon<\frac{\operatorname{inj} M^{n}}{2}$, where $\operatorname{inj} M^{n}$ is the injectivity radius of $M^{n}$, the following assertion holds: Provided there exists exactly one geodesic between $x$ and $y$ of length $\leq \bar{L}+(2 N-3) L+(2 N-2) \varepsilon$, then there exists a coherent $N$-filling of $(m, \varepsilon)$-umbrellas based at $x, z$ from $U_{m, \varepsilon, x, z}^{L, \bar{L}}$.

Proof. The proof is inductive on $N$. It follows the same pattern as the proof of Proposition 2.3. To prove the base of induction we define $\phi_{1}(u)=u$ for every 1umbrella $u$. Assume that the theorem holds for $N=m-1,(m>1)$. To prove 
the theorem for $N=m$ note that conditions 1 and 3 imply that we have no choice in construction of $\psi_{m}(u)=\left.\phi_{m}(u)\right|_{\partial \sigma^{m}}$ : One of $(m+1)$ faces of $\sigma^{m}$ of dimension $(m-1)$ must be mapped using the mapping $\rho$, whereas the remaining $m$ faces should be mapped using $\phi_{m-1}\left(u_{i}\right)$. Using a part of the induction assumption we can conclude that $\psi_{m}$ is a continuous function of $u$.

It remains only to contract $\psi_{m}(u)$ by a homotopy that continuously depends on $u$. The idea is to eliminate the simplex $\rho$ by contracting it over its image and then to proceed as in the proof of Proposition 2.3.

Recall that $s_{*}$ denotes the center of $\sigma^{m-1}$. Fix a contraction $h_{t}$ of $\sigma^{m-1}=$ $\left[s_{1}, s_{2}, \ldots, s_{m}\right]$ to $\left\{s_{*}\right\},\left(h_{0}\right.$ is the identity map, $\left.h_{1}\left(\sigma^{m-1}\right)=\left\{s_{*}\right\}\right)$, such that all points of $\sigma^{m-1}$ move to $S_{*}$ along straight lines with a constant speed. This will provide us with a homotopy of umbrellas: If $u=\left(c_{1}, \ldots, c_{m}, \rho\right)$, then $H_{t}(u)$ is defined as $\left(c_{1 t}, \ldots, c_{m t}, \rho \circ h_{t}\right)$, where $c_{i t}$ is the join of $c_{i}$ with $\rho\left(\left[s_{i} h_{t}\left(s_{i}\right)\right]\right)$ for every $i$. If $u$ is an $(m, \varepsilon)$-umbrella, the length of $c_{i t}$ does not exceed the sum of the length of $c_{i}$ and $\varepsilon$. For every $t \in[0,1]$ we can consider $\left.\psi_{m}\left(H_{t}(u)\right)\right)$. The composition $\psi_{m} \circ H_{t}$ will constitute the first stage in a homotopy contracting $\psi_{m}(u)$.

It remains to contract $\psi_{m}\left(H_{1}(u)\right)$. Note that $H_{1}(u)$ looks like an $m$-cage since its $(m-1)$-dimensional simplex is constant. Therefore we can contract the resulting $(m-1)$-dimensional sphere repeating the corresponding step in the proof of Proposition 2.3 almost verbatim.

Namely, we use Lemma 2.4 to construct a path homotopy $c_{1 t},(t \in[1,2])$, between $c_{11}=h_{1}\left(c_{1}\right)$ and $c_{21}=h_{1}\left(c_{2}\right)$ such that it passes only through paths of length $\leq \bar{L}+2 L+3 \varepsilon$. Let $u_{t}=\left(c_{t}, c_{21}, \ldots, c_{m 1}, \rho \circ h_{1}\right)$. The next stage of our homotopy contracting $\psi_{m}(u)$ will consist of $(m-1)$-dimensional spheres $\psi_{m}\left(u_{t}\right), t \in[1,2]$.

Finally, note that $u_{2}=\left(c_{21}, c_{21}, \ldots, c_{m 1}, \rho \circ h_{1}\right)$, so that $\phi_{m}\left(u_{2}\right)$ will be a "folded" $(m-1)$-dimensional sphere that can be canonically contracted over itself exactly as this had been done in the proof of Proposition 2.3.

\section{Proof of Theorem 1.1}

We are going to prove the theorem by contradiction. Assume that there exists exactly one geodesic between $x$ and $y$ of length $\leq 2 q d$. Therefore there exists $\delta>0$ such that there exists exactly one geodesic between $x$ and $y$ of length $\leq 2 q d+\delta$. (Indeed, otherwise there will be a sequence of geodesics between $x$ and $y$ with lengths strictly decreasing to $2 q d$. The Ascoli-Arzela theorem implies that a subsequence of this sequence converges to a geodesic between $x$ and $y$ of length $2 q d \geq 2 d>d$. Therefore, this geodesic cannot be minimizing and, therefore, is the second geodesic between $x$ and $y$ of length $\leq 2 q d$, which contradicts to our assumption.) Let $\varepsilon=$ $\min \left\{\frac{\delta}{2 n}, \frac{\operatorname{inj}\left(M^{n}\right)}{2}\right\}$, where $\operatorname{inj}\left(M^{n}\right)$ denotes the injectivity radius of $M^{n}$. Let $f: S^{q} \rightarrow$ $M^{n}$ be a non-contractible map of the $q$-dimensional sphere into $M^{n}$. 
We are going to extend $f$ to a map of the $(q+1)$-dimensional disc $D^{q+1}$ thereby reaching the desired contradiction. First, choose a fine smooth triangulation of $S^{q}$ such that that for every singular simplex $\tau: \sigma^{i} \rightarrow S^{q+1},(i \in\{1, \ldots, q+1\})$, the image under $f \circ \tau$ of $\sigma^{i}$ is contained in an $\varepsilon$-ball centered at the image of the center of $\sigma^{i}$ under $f \circ \tau$, and the length of the image of every straight line segment in $\sigma^{i}$ under $f \circ \tau$ is less than $\varepsilon$.

Triangulate $D^{q+1}$ as the cone over the chosen triangulation of $S^{q+1}$. Extend $f$ to the 1-skeleton of the triangulation of $D^{q+1}$ by mapping the center of $D^{q+1}$ to $x$, and every new 1-dimensional simplices into a minimal geodesic between the images of the endpoints of the 1-simplex. (Here one can choose any minimal geodesic, if there is more than one.)

We are going to continue the extension process inductively. Assume that we have already extended $f$ to the $i$-skeleton of the triangulation of $D^{q+1}$. In order to extend it to the $(i+1)$-skeleton observe that every new $(i+1)$-dimensional simplex is a cone over a $i$-dimensional simplex $\tau$ of the chosen triangulation of $S^{q}$. Consider a $(i+1, \varepsilon)$-umbrella based at $x$ and the image of the center of $\tau$ under $f$, such that $\rho=f \circ \tau$. Take $\bar{L}=L=d$. Apply Proposition 3.3 to fill this umbrella. The coherence condition implies that the resulting map of the $(i+1)$-dimensional simplex of the triangulation of $D^{q+1}$ extends maps of its faces constructed on the previous steps of the induction.

Once $f$ is extended to the $(q+1)$-skeleton of $D^{q+1}$, the extension process becomes complete, and we obtain the desired contradiction.

\section{Concluding remarks}

In [NR1] we made the following conjecture:

Conjecture 5.1. There exists a function $f(n, k)$ such that for every positive integer $k$, every closed Riemannian manifold $M^{n}$ and every pair of points $x, y \in M^{n}$ there exist $k$ distinct geodesics between $x$ and $y$ in $M^{n}$ of length $\leq f(n, k) d$, where $d$ denotes the diameter of $M^{n}$.

In fact, we made even a stronger conjecture that there exist $k$ distinct geodesics of length $\leq k d$. This stronger conjecture holds for round spheres and for all closed Riemannian manifolds with infinite torsion-free fundamental groups. Yet, F. Balacheff, C. Croke and M. Katz recently constructed Riemannian metrics on $S^{2}$ arbitrarily close to round metrics such that the length of the shortest geodesic loop at every point is strictly greater than $2 d$ ([BCK]). Thus, this stronger conjecture is not true even when $n=k=2$ and $x=y$.

In the present paper we proved our conjecture for $k=2$ for an arbitrary $M^{n}$ and arbitrary $x, y \in M^{n}$. Thus, we demonstrated that one can take $f(n, 2)=2 n$. 
Our paper [NR2] contains another result in this direction: If $n=2$ and $M^{n}$ is diffeomorphic to $S^{2}$, then for every $k$ and every pair of points $x, y$ in the Riemannian manifold there exist $k$ distinct geodesics between $x$ and $y$ of length $\leq\left(4 k^{2}-2 k-1\right) d$. Therefore, one can take $f(2, k)=4 k^{2}-2 k-1$.

Our most recent result in this direction establishes the conjecture for all Riemannian manifolds homotopy equivalent to the product of $S^{2}$ and an arbitrary closed manifold. In this case for every pair of points $x, y$ there exist at least $k$ distinct geodesics between $x$ and $y$ of length $\leq 20 k ! d$ (see [NR3]).

Acknowledgements. The work of the first author has been partially supported by his NSF Grants DMS-0706803 and DMS-0405954 as well as by by NSERC Discovery Grant. The work of the second author has been partially supported by her NSF Grant DMS-0604113, by NSERC Discovery Grants as well as by NSERC University Faculty Award.

\section{References}

[BCK] F. Balacheff, C. Croke, M. Katz, A Zoll counterexample to a geodesic length conjecture. Geom. Funct. Anal. 19 (2009), 1-10. Zbl 05575926

[C] C. Croke, Area and the length of the shortest closed geodesic. J. Differential Geom. 27 (1988), 1-21. Zbl 0642.53045 MR 0918453

[G] M. Gromov, Filling Riemannian manifolds. J. Differential Geom. 18 (1983), 1-147. Zbl 0515.53037 MR 0697984

[NR1] A. Nabutovsky and R. Rotman, Length of geodesics between two points on a Riemannian manifold. Electron. Res. Announc. Amer. Math. Soc. 13 (2007), 13-20. Zbl 1113.53026 MR 2285762

[NR2] A. Nabutovsky and R. Rotman, Lengths of geodesics on a two-dimensional sphere. Amer. J. Math. 131 (2009), 545-569. Zbl 05549758 MR 2503992

[NR3] A. Nabutovsky and R. Rotman, Short geodesic segments between two points on a closed Riemannian manifold. Geom. Funct. Anal., to appear; published online DOI 10.1007/ s00039-009-0004-8.

[R] R. Rotman, The length of a shortest geodesic loop at a point, J. Differential Geom. 78 (2008), 497-519. Zbl 1143.53038 MR 2396252

[S] J. P. Serre, Homologie singulière des espaces fibrés. Applications. Ann. of Math. 54 (1951), 425-505. Zbl 0045.26003 MR 0045386

Received November 7, 2007

Alexander Nabutovsky and Regina Rotman, Department of Mathematics, University of Toronto, Toronto, Ontario, M5S 2E4, Canada

E-mail: alex@math.toronto.edu; rina@math.toronto.edu 